\title{
Sostenibilidad: Una evaluación en las explotaciones agropecuarias conexas al Parque Nacional Natural Chingaza en el Municipio de San Juanito (Meta-Colombia)
}

\section{Sustainability: An evaluation on agricultural farms related to the Chingaza National Park in the Municipality of San Juanito (Meta-Colombia)}

\author{
CASTRO-GARZÓN, Hernando ${ }^{1}$ \\ MARTÍNEZ, Anderson D. ${ }^{2}$ \\ RODRÍGUEZ, Juan P. ${ }^{3}$
}

\begin{abstract}
Resumen
A través de una investigación cuantitativa aplicada a las explotaciones ubicadas en áreas conexas al Parque Nacional Chingaza, en el municipio de San Juanito, Meta, Colombia, se llevó a cabo una adaptación de la metodología propuesta por Sarandón (2009) para evaluar la sustentabilidad de 37 predios en 5 veredas del municipio. Se aplicaron entrevistas para la recolección de información con la que se construyeron indicadores para obtener el índice general de sustentabilidad.

Palabras clave: áreas protegidas, evaluación de sustentabilidad, multicriterio, sostenible.
\end{abstract}

\begin{abstract}
Through quantitative research applied to farms located in areas related to Chingaza National Park, in the municipality of San Juanito, Meta, Colombia, an adaptation of the methodology proposed by Sarandón (2009) was carried out to assess the sustainability of 37 sites on 5 sidewalks of the municipality. Interviews were applied for the collection of information with which indicators were built to obtain the overall sustainability index.
\end{abstract}

Key words: protected areas, sustainability assessment, multicrime, sustainable.

\section{Introducción}

Colombia con una superficie total de más de un millón ciento cuarenta mil kilómetros cuadrados de los cuales el $8.8 \%$ es agua (Ministerio de Comercio Industria y Turismo, 2018) y el $11,3 \%$ son áreas protegidas, las cuales están administradas y supervisadas por el Sistema Nacional Áreas Protegidas (SINAP) el cual agrupa este porcentaje en un total de 59 áreas protegidas (Vásquez M \& Andrade, 2016, p.129).

\footnotetext{
${ }^{1}$ Profesor, Escuela de Administración y Negocios. Facultad de Ciencias Económicas, Universidad de los Llanos, Villavicencio, Colombia. Correo electrónico: hcastro@unillanos.edu.co

${ }^{2}$ Administrador de Empresas. Universidad de los Llanos, Villavicencio, Colombia. Correo electrónico:

anderson.martinez@unillanos.edu.co

${ }^{3}$ Profesor Titular. Facultad del Medio Ambiente y Recursos Naturales. Universidad Distrital Francisco José de Caldas. Bogotá, Colombia. Correo electrónico: jprodriguezm@udistrital.edu.co
} 
El modelo de conservación planteado por los gobiernos es altamente restrictivo en cuanto al uso y manejo de los recursos, proponiendo reglas que establecen quién, cómo, cuándo y dónde puede usarlos (Adams \& Hutton, 2007, P.150), esta función desarrollada en Colombia por el SINAP instaura la conservación como única actividad permitida dentro de las Áreas de Protección (AP) (Parques Nacionales Naturales de Colombia, 2015) coaccionando a los habitantes de estas zonas en el desarrollo de sus actividades económicas, culturales y sociales. De esta manera se genera un conflicto en la declaración de AP puesto que la interacción de ser humanonaturaleza se ve interrumpida por las políticas de conservación, así lo permite observar Gutiérrez Antolínez (2016, p.43).

El mecanismo de sustraer un territorio como área protegida se hacía hasta el siglo pasado teniendo en cuenta solo criterios científicos y de altura, tales como territorios rurales superior a los $1800 \mathrm{msnm}$ y la conservación de la biodiversidad del lugar, no se valoraba la presencia de habitantes en el lugar y la afectación a sus actividades productivas. Esta visión cambio en el 2003 durante el -V congreso mundial de Parques Nacionales y Áreas Protegidas- en donde el involucramiento del factor humano comienza a hacer parte de los ejes de planeación y conservación de estas zonas (Jenkins \& Joppa, 2009, p.2167).

Así las cosas, para las administraciones de los gobiernos AP son objeto de normalización sin afectación a la población, permitiendo desarrollar estrategias de involucramiento de los agentes y actores acercando las políticas de conservación y desarrollo rural, tal es el caso de San Mateo Almomoloa en el Estado de México (Cruz Moreno \& Zizumbo Villarreal, 2017, p.944) en donde la financiación y asesoramiento de proyectos productivos se hace a través del gobierno con y para la comunidad, robusteciendo actos que repercuten en el desarrollo local.

Hay que mencionar además el apoyo económico que pueden llegar a tener estas AP por el pago de servicios ambientales, permitiendo la conservación, cuidado ambiental y cultural de dichas áreas; así como sucede en Salta Argentina (Barbarán \& Rojas, 2015, p.23), Bosques templados de México (Galicia, et al., 2018), Costa Rica como pionero mundial (Sánchez-Chaves \& Navarrete-Chacón, 2017), donde las comunidades campesinas, indígenas y afrodescendientes tienen la oportunidad de dar un uso apropiado a los recursos tales como el suelo, el agua, el aire indispensables para su sostenimiento sin alterarlos.

El presente trabajo pretende mostrar en términos de sostenibilidad de las explotaciones agropecuarias presentes en el municipio de San Juanito, Meta, Colombia; a través de índices obtenidos para la apreciación cuantitativa de los diferentes indicadores generados a través de la observación y el procesamiento de datos obtenidos por medio de la herramienta diseñada.

\section{Metodología}

\subsection{Descripción del Lugar}

Para este estudio, se tuvieron en cuenta 37 unidades productivas del municipio San Juanito, distribuidas en 5 de sus 9 veredas. Los predios analizados tienen tamaños entre 8 y 24 Has. En ellos se realizan actividades agropecuarias: cultivos principalmente de (fríjol, maíz y caña); producción de ganado bovino y en menos proporción actividades de transformación de leche y panela.

\subsection{Construcción de Indicadores}

Los indicadores que se crearon siguen la metodología propuesta por (Saradón, 2009, p.23). Se seleccionaron indicadores fáciles de recolectar y tabular, que brindan información relevante para cada una de las dimensiones del desarrollo sostenible. Estos indicadores se dividieron en subindicadores para generar una ponderación que incluyera diferentes variables por cada dimensión. Los datos provienen de una encuesta realizada en campo durante el segundo semestre del 2017 que fue posteriormente tabulada y anonimizada. 
Para poder realizar una comparación entre las diferentes unidades productivas, se estandarizaron las variables en una escala para los indicadores de 1 a 5 , siendo 1 el valor más bajo de sostenibilidad y 5 el mayor. Luego, se ponderaron los indicadores teniendo en cuenta la importancia de cada variable respecto a la sostenibilidad.

\subsection{Indicador para la dimensión económica}

Para determinar si las unidades productivas eran sostenibles económicamente, se eligieron los siguientes subindicadores.

Seguridad Alimentaria: Se consideró que la autosuficiencia alimentaria es importante para determinar la sostenibilidad, para calcularla se utilizaron los subindicadores de:

a. Diversificación de cultivos: un sistema es sostenible si la producción esta diversificada y se evaluó así: un producto o menos (1), entre 2 y 4 productos (2), entre 4 y 6 productos (3), entre 6 y 10 productos (4), más de 10 productos $(5)$

b. Área para autoconsumo: un sistema es sostenible si el área destinada para autoconsumo es suficiente para satisfacer el consumo de los integrantes del hogar. Esta variable se clasifico así: menos de 0.5 ha (1), entre 0.05 y 0.5 ha (2), entre 0.5 y 1 ha (3), entre 1 y 2 ha (4), más de 2 ha (5)

Ingresos del Hogar: un sistema es sostenible si puede satisfacer sus necesidades económicas, los ingresos se clasificaron en salarios mínimos legales vigentes: menos de 1 SMLV (1), un SMLV (2), entre 1 y 2 SMLV (3), entre 2 y 4 SMLV (4), más de 4 SMLV (5).

1. Riesgo Económico. Un sistema es sostenible cuando puede diversificar el riesgo económico y asegurar la estabilidad de la producción. Este indicador se subdividió en:

a. número de canales de comercialización: un canal (1), 2 canales (2), 3 canales (3), 4 canales (4), 5 o más canales (5).

b. Dependencia de insumos externos: un sistema con poca dependencia de insumos externos es sostenible en el tiempo. Se categorizo como sigue: de 80 a $100 \%$ de insumos externos (1); de 60 a $80 \%$ de insumos externos (2); de 40 a $60 \%$ de insumos externos (3); de 20 a $40 \%$ de insumos externos (4); de 0 a $20 \%$ de insumos externos (5).

El indicador de sostenibilidad económica se calcula con la fórmula:

$$
\text { DimensiónEconómica }=\frac{\frac{1 \cdot a+1 \cdot b}{2}+2 \cdot a+\frac{3 \cdot a+3 \cdot b}{2}}{3}
$$

\subsection{Indicador para la dimensión ambiental}

Esta dimensión, se evaluó teniendo en cuenta los siguientes subindicadores:

Conservación del suelo: un sistema es sostenible si las actividades que se realizan en el suelo buscan mantener o mejorar sus características. Para calcularlo se utilizaron:

a. Manejo de la cobertura vegetal. Esta protege el suelo de los rayos directos del sol y reduce el riesgo de erosión. Dependiendo del nivel de cobertura se categorizo en: menos del $25 \%$ (1), entre 25 y $50 \%$ (2), entre 50 y $75 \%(3)$, entre 75 y $99 \%$ (4), 100\% (5). 
b. Rotación de cultivos. No realiza rotación (1), Hace rotaciones eventualmente (2), Rota casa 2 o 3 años (3), Rota todos los años. No deja descansar el suelo (4), rota todos los años. Deja descansar el suelo, abona o siembras leguminosas (5).

c. Riesgo de erosión. Disminuir el riesgo de pérdida de suelos por erosión es importante para la sostenibilidad. Para esta variable Se tuvo en cuenta:

d. Pendiente principal del predio: Mayor de $40^{\circ}(1)$, entre 27 y $40^{\circ}(2)$, entre 13 y $27^{\circ}$ (3), entre 4.5 y $13^{\circ}$ (4), menos de $4.5^{\circ}(5)$.

e. Orientación de los surcos al sembrar: Surcos paralelos a la pendiente (1), Surcos orientados $30^{\circ}$ con respecto a la pendiente (2), surcos orientados $60^{\circ}$ con respecto a la pendiente (3), surcos perpendiculares a la pendiente (4), curvas de nivel o terrazas (5). Este subindicador tuvo un peso doble en la ponderación.

Con lo anterior, la fórmula para calcular el indicador de sostenibilidad ambiental es:

$$
\text { DimensiónAmbiental }=\frac{\frac{1 \cdot a+1 \cdot b}{2}+\frac{2 \cdot a+2(2 \cdot b)}{3}}{2}
$$

\subsection{Indicador para la dimensión social}

La satisfacción social se evaluó mediante:

Satisfacción de las necesidades básicas. Un sistema es sostenible si los integrantes de hogar han resuelto sus necesidades básicas como vivienda, educación, salud y servicios. Se le dio un peso doble en la ponderación.

a) Vivienda, material predominante de las paredes: Bloque o ladrillo (5), Adobe (4), Madera, tablón, otros vegetales (3), Latas, zinc, plástico (2), otros materiales (1).

b) Vivienda, material predominante de los pisos: Baldosa (Mármol, cerámica o piedra) (5), madera (4), ladrillo (3), cemento (2), tierra o arena (1).

c) Acceso a educación: Instituciones de educación superior (5), educación secundaria (4), educación primaria (3), educación secundaria y primaria con limitaciones (2), sin acceso a educación (1).

d) Acceso a salud: Centro sanitario con médicos fijos e infraestructura adecuada (5); Centro sanitario con personal temporal y medianamente equipado (4); Centro sanitario con personal temporal y mal equipado (3); Centro sanitario sin personal y mal equipado (2); Sin centro sanitario (1).

e) Acceso a servicios públicos: Electricidad, acueducto y teléfono (5); Electricidad y acueducto (4); Electricidad y agua mediante fuente cercana (3); Sin electricidad y agua mediante fuente cercana (2), Sin servicios (1).

Aceptabilidad del sistema de producción.

a) Se preguntó directamente por el grado de satisfacción: Está muy contento con lo que hace. No haría otra actividad, aunque ésta le reporte más ingresos (5); Está contento, pero antes le iba mucho mejor (4); No está del todo satisfecho. Se queda porque es lo único que sabe hacer (3); Poco satisfecho con esta forma de vida. Anhela vivir en la ciudad y ocuparse de otra actividad (2); Está desilusionado con la vida que lleva. Está esperando que se le presente una oportunidad para dejar la producción (1).

Con lo anterior, la fórmula para calcular el indicador de sostenibilidad social es:

$$
\text { DimensiónSocial }=\frac{2 \frac{(1 \cdot a+1 \cdot b+1 \cdot c+1 \cdot d+1 \cdot e)}{5}+2 \cdot a}{3}
$$


Índice General de Sostenibilidad

$$
\text { ÍndiceGeneralSostenibilidad }=\frac{\text { DimensiónEconomica }+ \text { DimensiónAmbiental }+ \text { DimensiónSocial }}{3}
$$

Al igual que en (Saradón, 2009) se definió un valor umbral o mínimo que debía alcanzar el índice de sostenibilidad general (ISG), para considerar un predio sostenible: igual o menor que el valor medio de la escala, es decir, 3. Además, se consideró que ninguna de las 3 dimensiones debía tener un valor menor a 2.5. Para todos los cálculos se usó «R (Versión 3.5.1; R Core Team, 2018) y los paquetes dplyr (Versión 0.7.5; Wickham, et al, (2018).

\section{Resultados}

Tabla 1

Valores de los Indicadores para los 37 Predios

\begin{tabular}{|c|c|c|c|c|c|}
\hline Cod. Predio & Dim. Económica & Dim. Ambiental & Dim. Social & Índice General & Sostenible \\
\hline 3002 & 2,33 & 1,75 & 2,6 & 2,23 & NO \\
\hline 3208 & 2,33 & 1,75 & 2,73 & 2,27 & NO \\
\hline 2107 & 2,5 & 1,92 & 2,47 & 2,29 & NO \\
\hline 1024 & 2,33 & 1,75 & 2,87 & 2,32 & NO \\
\hline 1010 & 2,5 & 2 & 2,67 & 2,39 & NO \\
\hline 1005 & 2 & 3 & 2,53 & 2,51 & NO \\
\hline 3205 & 2,17 & 2,92 & 2,67 & 2,58 & NO \\
\hline 1022 & 1,67 & 3,58 & 2,53 & 2,59 & NO \\
\hline 2111 & 2,17 & 2,67 & 3 & 2,61 & NO \\
\hline 3204 & 2,33 & 1,75 & 4,07 & 2,72 & NO \\
\hline 2110 & 2,17 & 3,25 & 2,87 & 2,76 & NO \\
\hline 1015 & 2,17 & 3,42 & 3 & 2,86 & NO \\
\hline 2106 & 2,17 & 3,25 & 3,33 & 2,92 & NO \\
\hline 2102 & 2,17 & 4,08 & 2,87 & 3,04 & NO \\
\hline 3201 & 2,33 & 3,33 & 3,47 & 3,04 & NO \\
\hline 3212 & 2,33 & 3,42 & 3,47 & 3,07 & NO \\
\hline 3211 & 2 & 3,17 & 4,07 & 3,08 & NO \\
\hline 3206 & 2,33 & 3,33 & 3,67 & 3,11 & NO \\
\hline 2105 & 2 & 3,42 & 3,93 & 3,12 & NO \\
\hline 3203 & 2,33 & 3,08 & 3,93 & 3,12 & NO \\
\hline 1003 & 2 & 4 & 3,4 & 3,13 & NO \\
\hline 1007 & 2,5 & 4,08 & 2,87 & 3,15 & SI \\
\hline 3207 & 2 & 3,42 & 4,07 & 3,16 & NO \\
\hline 2109 & 1,83 & 3,75 & 4,07 & 3,22 & NO \\
\hline 1002 & 2,33 & 3,25 & 4,07 & 3,22 & NO \\
\hline 2108 & 2 & 3,75 & 4,07 & 3,27 & NO \\
\hline 1018 & 2,33 & 3,67 & 3,87 & 3,29 & NO \\
\hline 1019 & 2,33 & 3,25 & 4,33 & 3,31 & NO \\
\hline 3209 & 2,67 & 3,75 & 3,8 & 3,41 & SI \\
\hline 3210 & 2,67 & 3,17 & 4,47 & 3,43 & SI \\
\hline 2104 & 2,17 & 3,75 & 4,47 & 3,46 & NO \\
\hline 2101 & 2,33 & 4 & 4,07 & 3,47 & NO \\
\hline 1008 & 3,17 & 3,67 & 3,8 & 3,54 & SI \\
\hline 1004 & 2,67 & 4,42 & 3,67 & 3,58 & SI \\
\hline 1012 & 2,5 & 4,25 & 4,07 & 3,61 & SI \\
\hline 1006 & 2,5 & 4,08 & 4,73 & 3,77 & SI \\
\hline 1016 & 3,17 & 4,08 & 4,33 & 3,86 & SI \\
\hline Total & 2,31 & 3,27 & 3,54 & 3,04 & NO \\
\hline
\end{tabular}




\subsection{Análisis de Sostenibilidad}

Los indicadores construidos brindan información que permite contrastar los resultados de todos los predios, que a pesar de estar en un mismo municipio y tener prácticas y cultivos similares, presentan indicadores de sostenibilidad variados. El promedio del índice general de sostenibilidad fue superior al umbral (3.04), pero en la dimensión económica (2.31) no supero el valor mínimo para cada dimensión por lo cual en general los 37 predios analizados en el municipio según esta metodología, no cumplen la condición para considerarse sostenibles.

En total, solo 8 predios cumplen con los requisitos de sostenibilidad, y solo una de las 5 veredas analizadas puede considerarse sostenible. La dimensión mejor evaluada fue la dimensión social (3.54), seguido de la Ambiental (3.27) los subíndices que más influyeron en estos resultados fueron la aceptación del sistema productivo y la conservación del suelo.

En materia de sostenibilidad económica, es donde el municipio tiene mayor deficiencia. El subíndice peor evaluado fue la seguridad alimentaria con 1.8 (Figura. 1), esto se puede explicar principalmente por la marcada dependencia de cultivo de fríjol en el municipio. La rentabilidad de este cultivo ha desplazado la producción de los demás alimentos por lo que hace a los hogares muy dependientes de alimentos e insumos externos. El riesgo económico también está por debajo del umbral, principalmente porque la mayoría de las fincas solo utilizan un canal de comercialización (Intermediario).

Figura 1

Diseño en tela de araña para los subíndices

SegAlimentaria.E

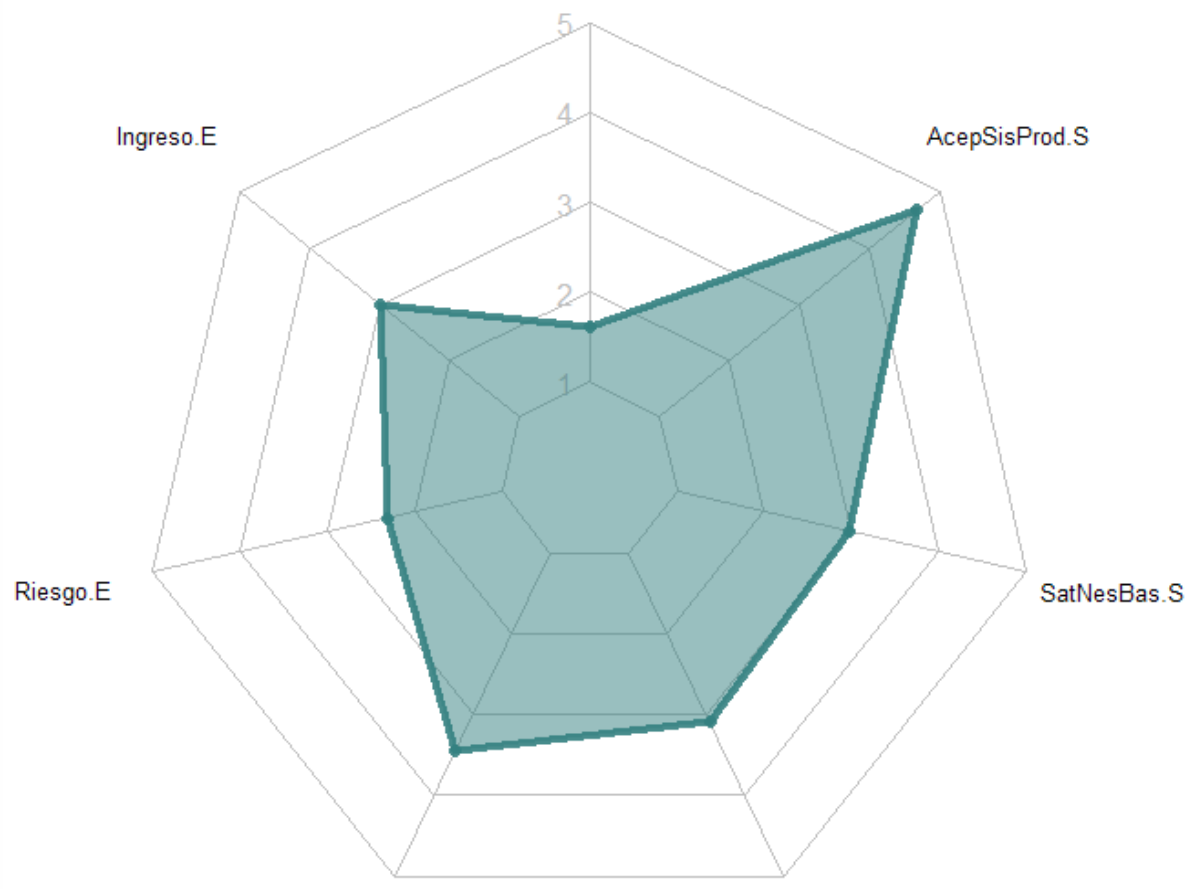

ConserSuelo.A

RiesgoErosi.A

Agregando la información por vereda (Tabla 2), se encontró que 3 de las 5 evaluadas no superan el umbral para el índice general. En el caso de La Candelaria, esta no cumple en ninguna de las 3 dimensiones. Las otras 2 superan el umbral, pero San Luis El Plan no cumple con la dimensión económica y por lo tanto no satisface la condición para ser sostenible; Únicamente El Tablón con 2.69, 3.58 y 4.05 en promedio, respectivamente para cada dimensión y con un IGS de 3.44 aprueba los requisitos para ser considerada una vereda sostenible. 
Tabla 2

Valores de los Indicadores Para las Veredas Analizadas

\begin{tabular}{l|c|c|c|c|c}
\hline \multicolumn{1}{c}{ Vereda } & Dim. Económica & Dim. Ambiental & Dim. Social & Índice General & Sostenible \\
\hline La Candelaria & 2.19 & 2.82 & 2.78 & 2.60 & NO \\
\hline San Luis de Toledo & 2.17 & 3.15 & 3.42 & 2.91 & NO \\
\hline El Carmen & 2.36 & 3.21 & 3.26 & 2.94 & NO \\
\hline San Luis El Plan & 2.19 & 3.50 & 3.97 & 3.22 & 3.44 \\
\hline El Tablón & 2.69 & 3.58 & 4.05 & SI \\
\hline
\end{tabular}

Fuente 1 Elaborado por los autores. (2019)

Figura 2

Mapa Índice General y Dimensiones por Veredas

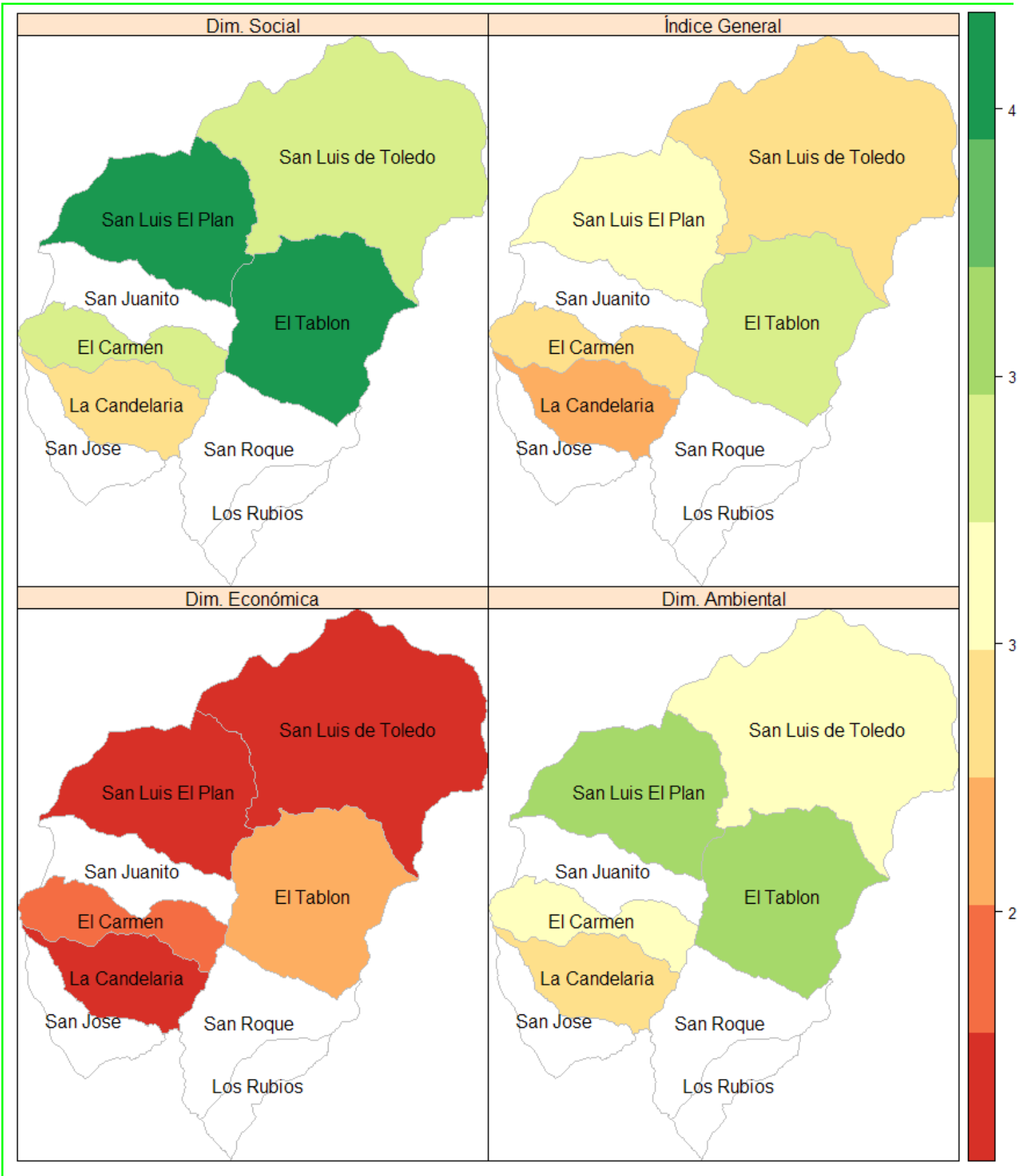

Fuente: Elaborado por los autores (2019) 
Un análisis de las dimensiones por veredas, permite identificar que dimensiones están mejor que otras y que ubicaciones. Esto permite dar recomendaciones de política social, económica y ambiental focalizada para la comunidad de cada vereda. Es importante resaltar que en el ámbito ambiental 4 de las 5 veredas cumplen con el umbral. Lo anterior teniendo en cuenta que el municipio está ubicado dentro del área protegida y en la zona de amortiguación del parque nacional natural Chingaza. En general, las prácticas de conservación del suelo y de mitigación del riesgo de erosión presentan resultados positivos.

\subsection{Discusión}

Los resultados obtenidos mediante esta metodología reflejan que las actividades agropecuarias realizadas en el municipio de San Juanito Meta en general no son sustentables debido principalmente a la dimensión Económica, es decir por falta de diversificación y de canales de comercialización los productores se enfrentan a un alto riesgo económico al depender en su mayoría de un solo producto (frijol) y un solo canal (intermediario). Por otra parte, esto también afecta la seguridad alimentaria agregando que el área destinada a autoconsumo es significativamente baja y no alcanza un mínimo para garantizar la alimentación de los habitantes en un caso de fuerza mayor en que quedase incomunicado por vía terrestre.

Por otra parte, en los predios entrevistados en la zona de paramo, más específicamente la zona denominada de transición, se encontró que la actividad principal es la ganadería, a pesar de no ser intensiva y que el promedio es de 0.6 cabezas por hectárea, se puede apreciar que esta actividad no es sostenible en ninguna de las dimensiones analizadas:

En cuanto a la parte económica no es rentable debido al bajo nivel de producción, al riesgo económico y la pérdida de animales el cual se presenta constantemente debido a los ataques de osos en la zona, al costo de transporte hasta el lugar del predio: teniendo en cuenta que no hay vías de acceso y el trayecto se realiza caminando en tiempos que rondan de 3 a 10 horas por pendientes que superan los $50^{\circ}$.

Desde la dimensión social, teniendo en cuenta lo mencionado anteriormente sobre las condiciones de acceso a los predios a esto debe sumarse que en la zona de paramo no se cuentan con servicios públicos de ningún tipo. Por lo que es completamente inhabitable, como prueba de ello, se tiene que en ninguno de los predios entrevistados de paramo se cuenta con instalaciones de vivienda.

Por último, en cuanto a la dimisión ambiental, existen numerosos estudios que han expuesto el tema. Estupiñán (2009) afirma: "la cría de ganado, específicamente vacuno, ha dejado una devastadora y aún no calculada pérdida de las condiciones de páramo, reflejado, especialmente, en la capacidad de retención de agua en el suelo que se ve afectada por el pisoteo constante del ganado, compactándolo y quitándole espacio poroso para acumular el agua" (p80).

\section{Conclusiones}

Evaluar la sostenibilidad es un reto difícil de alcanzar teniendo en cuenta la complejidad y la subjetividad del término. Usar indicadores mediante el método multicriterio resulta un ejercicio valido para abordar esta complejidad en términos de objetivos, cuantificar y poder comparar. De todas formas, no existen unos indicadores establecidos que sean universales para evaluar sostenibilidad por lo que los indicadores construidos están basados en la propuesta de (Sarandón, 2009) y adaptados al trabajo de campo realizado.

En el municipio de San Juanito no se ha realizado un estudio similar al que se expuso en esta investigación puesto que no es posible contrastar los resultados obtenidos. Sin embargo, se ha conseguido como resultado de este trabajo establecer unos indicadores de línea base para futuras investigaciones. Dado el índice de sostenibilidad tan bajo en la dimensión Económica, es necesario que se adelanten programas en seguridad alimentaria que 
garanticen el abastecimiento de productos agrícolas en caso de un cierre en sus vías de acceso. También se recomienda diversificar los cultivos en cada finca debido a que en su mayoría solo siembran entre uno y dos productos lo cual genera un mayor riesgo económico.

\section{Referencias bibliográficas}

Adams, W. M., \& Hutton, J. (2007). People, parks and poverty: political ecology and biodiversity conservation. Conservation and society, 5(2), 147-183.

Barbarán, F. R., \& Rojas, L. (2015). Sostenibilidad institucional y social de la expansión de la frontera agropecuaria. Boom sojero, políticas redistributivas y pago por servicios ambientales en el norte de Salta , Argentina. Revista Iberoamericana de Economía Ecológica, 24, 21-37.

Cruz Moreno, I. A., \& Zizumbo Villarreal, L. (2017). Alcances De La Política Social Y El Turismo Rural Para El Desarrollo Local En San Mateo Almomoloa -- México. Estudios y Perspectivas En Turismo, 26(4), 944-963. Retrieved from http://ezproxy.eafit.edu.co/login?url=http://search.ebscohost.com/login.aspx?direct=true\&db=fua\&AN=1 25512569\&lang=es\&site=eds-live \&scope=site

Estupiñan, L. H. (2009). Efecto de actividades agropecuarias en las características del suelo en el páramo El Granizo. Revista UDCA Actualidad \& Divulgación Científica, 79-89.

Galicia, L., Chávez-Vergara, B. M., Kolb, M., Jasso-Flores, R. I., Rodríguez-Bustos, L. A., Solís, L. E., \& Villanueva, A. (2018). Perspectivas del enfoque socioecológico en la conservación, el aprovechamiento y pago de servicios ambientales de los bosques templados de México. Madera y bosques, 24(2). https://doi.org/10.21829/myb.2018.2421443

Gutiérrez Antolínez, C. (2016). Conflictos socioambientales derivados de la declaración del Parque Nacional Natural Chingaza en zonas de producción campesina. Universidad Nacional de Colombia Facultad de Ciencias Económicas. Retrieved from http://bdigital.unal.edu.co/55505/1/carolinagutierrezantolinez.2016.pdf

Jenkins, C. N., \& Joppa, L. (2009). Expansion of the global terrestrial protected area system. Biological Conservation, 142(10), 2166-2174. https://doi.org/10.1016/J.BIOCON.2009.04.016

Ministerio de Comercio Industria y Turismo. (2018). Plan Nacional Sectorial del Turismo 2018 -2022. Bogotá. Retrieved from https://www.academia.edu/37607682/Plan_Nacional_Sectorial_del_Turismo_2018_-2022

Parques Nacionales Naturales de Colombia. (2015). Áreas Protegidas: Territorios para la Vida y la Paz. Tomo I: Áreas protegidas para el desarrollo. Retrieved from http://www.parquesnacionales.gov.co/portal/wpcontent/uploads/2015/11/PNNC_TOMO1_VF2.pdf

R Core Team. (2018). R: A Language and Environment for Statistical Computing. Vienna,Austria: R Foundation for Statistical Computing. Retrieved from https://www.r-project.org/

Sarandón, S. J., \& Flores, C. C. (2009). Evaluación de la sustentabilidad en agroecosistemas: una propuesta metodológica. Agroecología, 4, 19-28.

Sánchez-Chaves, O., \& Navarrete-Chacón, G. (2017). La experiencia de Costa Rica en el pago por servicios ambientales: 20 años de lecciones aprendidas. Revista de Ciencias Ambientales, 51(2), 195. https://doi.org/10.15359/rca.51-2.11

Vásquez M, J. L., \& Andrade, G. I. (2016). El paisaje protegido, pieza faltante en el Sistema de Áreas Protegidas de Colombia Resumen. Biodiversidad En La Práctica Documentos de Trabajo Del Instituto Humboldt, 1(2015), 128-146. 
Wickham, H., François, R., Henry, L., \& Müller, K. (2018). Dplyr: A grammar of data manipulation. Retrieved from https://CRAN.R-project.org/package=dplyr 